\title{
Investigation of seemingly pathogen-negative diarrhoea in patients infected with HIV1
}

\author{
G M Connolly, A Forbes, B G Gazzard
}

\begin{abstract}
Thirty three consecutive patients infected by human immunodeficiency virus type 1 (HIV1) with persistent diarrhoea which remained undiagnosed after microbiological examination of six stool samples and rectal histology were investigated for malabsorption. All had xylose and Schilling tests, distal duodenal biopsy, comprehensive barium studies, microbiological examination of six further stool samples, and repeat rectal histology. A microbiological or histological diagnosis of infection was made in 12 patients (multiple organisms in three). Cryptosporidia were identified on five occasions, cytomegalovirus on four, Giardia lamblia on two, and herpes simplex, Campylobacter jejuni, Salmonella enteritidis, and Entamoeba histolytica once each. No organism was found when weight loss was less than $5 \mathrm{~kg}$ or stool volume less than $400 \mathrm{ml} /$ day $(n=9)$. Pathogens were identified in nine of 13 patients $(69 \%)$ with weight loss greater than 10 $\mathrm{kg}$ and stool volume more than $800 \mathrm{ml} /$ day. Barium studies were normal except for ileal flocculation in two patients with cryptosporidiosis. Evidence for malabsorption existed in 24 patients - impaired xylose absorption $(n=19)$ and abnormal Schilling test $(n=21)$. Of the patients with a severely abnormal Schilling test, a pathogen was identified in $11(79 \%)$ (including all five with cryptosporidia, and two of the patients with only moderate diarrhoea and weight loss). A simple scoring system based on degree of weight loss and Schilling test result may help to identify the HIV positive patient with seemingly pathogen-negative diarrhoea in whom further investigations are likely to show a specific cause.
\end{abstract}

Diarrhoea is a common symptom in AIDS patients and occurs in up to $50 \%$ at some time during their illness. ${ }^{1}$ In a proportion of patients no pathogenic cause of their diarrhoea is found, and it has been hypothesised that direct human immunodeficiency virus type 1 (HIVl) infection of the gut mucosa leading to malabsorption may be responsible. ${ }^{2}$ The frequency of finding a pathogen obviously increases with the number of tests performed but the clinician needs advice to decide when further investigation is unlikely to yield diagnostic information. We prospectively studied a group of 33 patients in whom no pathogen was detected despite examination of six stool samples and rectal biopsy specimen. Further microbiological analysis, histopathology, and tests of malabsorption were performed to see if it was possible to predict which patients would benefit from these tests.

\section{Patients}

Thirty three consecutive male patients (median age 28 years; range $18-54$ ) with serum antibodies to HIV1 and with undiagnosed diarrhoea were studied. All had passed more than three loose stools daily for at least one month, with no microbiological diagnosis established from rectal histology and at least six stool examinations performed in a microbiology laboratory accustomed to the particular pathogens associated with this patient population. The CDC criteria for full AIDS $^{3}$ were fulfilled by 16 of the 33 patients at the time of presentation (group IV $\mathrm{Cl}$ in each case); the remaining 17 (by definition) had HIV disease group IV A.

\section{Methods}

Daily stool volume (mean of three days) and estimated weight loss from usual premorbid weight (checked against ideal 'weight for height' in cases of premorbid obesity or doubt) were recorded. Upper gastrointestinal endoscopy for distal duodenal biopsy, barium small bowel follow through examination, and double contrast barium enema were performed using standard methods.

Serum concentrations of vitamin B-12 and serum and red cell folate concentrations were measured, and double labelled cobalt Schilling tests carried out (normal greater than 11\% excreted). Xylose absorption testing ( $5 \mathrm{~g}$ ) was performed with assay of a five hour urine collection (normal greater than $1.4 \mathrm{~g}$ excreted).

Six further stool samples were examined microbiologically with special attention to opportunistic pathogens; accordingly the modified Ziehl-Nielsen stain and use of both LowensteinJensen and Kirchner media were used routinely as previously described. ${ }^{45}$

Rectal and duodenal histology were stained with haematoxylin and eosin, and with ZiehlNielsen stain. Inclusion bodies and an inflammatory response were required for a histological diagnosis of cytomegalovirus infecton.

Receiver operating characteristics curves $^{6}$ were constructed for each of the precisely quantifiable parameters measured. Sensitivity, specificity, and predictive values were defined and calculated conventionally.

A scoring system based on Bayes' theorem was developed to assist early identification of those in whom an infective aetiology for diarrhoea would eventually be found.

\section{Results}

Stool volume varied from $300 \mathrm{ml}$ to more than $3000 \mathrm{ml}$ per 24 hours. Use of arbitrary cut off points at 400 and $800 \mathrm{ml}$ led to division of 
Scattergram to show relation between xylose excretion, Schilling test result, and final evidence for or against infective aetiology for diarrhoea. Xylose results are excretion in $\mathrm{g} / 5 /$ hour urine collection and Schilling results are presented as the percentage of radioisotope excretion; in both cases the horizontal line marks the lower end of the normall reference range.

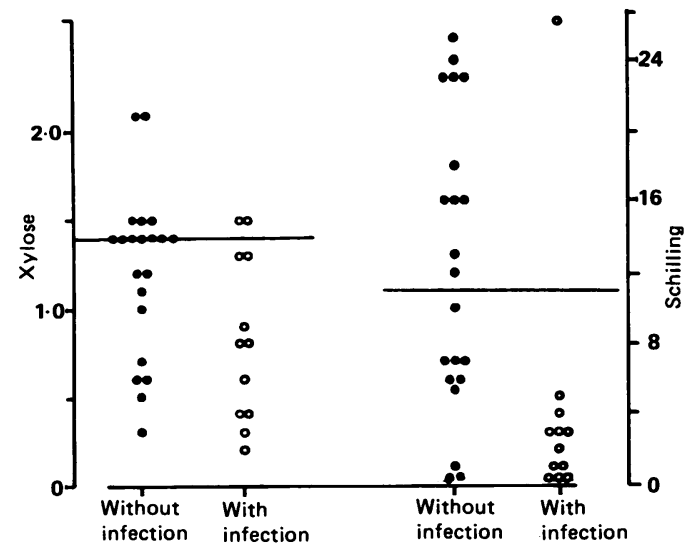

patients between three approximately equal sized groups: less than $400 \mathrm{ml}, \mathrm{n}=9 ; 400-800 \mathrm{ml}$, $\mathrm{n}=11$; more than $800 \mathrm{ml}, \mathrm{n}=13$. Similarly arbitrary cut off points for weight loss led to distribution of patients between three groups: less than $5 \mathrm{~kg}$ loss, $\mathrm{n}=9 ; 5-10 \mathrm{~kg}, \mathrm{n}=10$; more than $10 \mathrm{~kg}, \mathrm{n}=14$. The groupings form natural pairs and in all but one patient the stool volume grouping was 'equivalent' to that for weight loss.

Serum B-12 and folate and red cell folate concentrations were normal in all patients. Xylose excretion, however, was impaired in 19 patients and in nine of these excretion was less than $50 \%$ of the lower limit of normal (severely abnormal) (Figure). Unsurprisingly, the Schilling tests showed no evidence of intrinsic factor deficiency, and both labels were detected at similar radioconcentration: the mean of the two values for each patient was therefore used. In 21 patients abnormal values were detected and in 14 of these, mean values of less than $50 \%$ of the lower limit of normal were recorded (severely abnormal).

Infection was finally shown in 12 patients (more than one organism in three): cryptosporidium, n=5; Giardia lamblia, $\mathrm{n}=2$; Entamoeba histolytica, $\mathrm{n}=1$; Campylobacter jejuni, $\mathrm{n}=1$; Salmonella enteritidis, $\mathrm{n}=1$; cytomegalovirus, $\mathrm{n}=4$; and herpes simplex virus, $\mathrm{n}=1$. Diagnosis of cytomegalovirus was from biopsy specimen alone. Diagnosis of herpes simplex virus was from biopsy specimen and from swabs taken from rectal vesicles. Cryptosporidium was detected in both biopsy specimen and stool microbiology in two patients. The remaining diagnoses were from stool microbiology alone. All histological diagnoses were obtained from rectal biopsy specimens except for one patient where rectoscopy was normal but typical cytomegalovirus ulcers were seen in the transverse colon at colonoscopy and a biopsy confirmed cytomegalovirus infection.

\section{CORRELATION OF MALABSORPTION AND} INFECTION

An estimated weight loss of more than $10 \mathrm{~kg}$ was strongly associated with eventual identification of gastrointestinal pathogens: nine of 14 patients with this extent of weight loss had organisms detected, accounting for nine of the 12 patients in whom an organism was eventually found. When weight loss was less than $5 \mathrm{~kg}$ or daily stool volume was under $400 \mathrm{ml}$, or both, no pathogens
Sensitivity, specificity, and positive predictive value (PPV) for each of the degrees of abnormality of the parameters evaluated, with negative predictive value (NPV) for a normal result (percentages in each case).

\begin{tabular}{lcccc}
\hline & Sensitivity & Specificity & $P P V$ & $N P V$ \\
\hline Weight loss: & & & & \\
$>5 \mathrm{~kg}$ & 100 & 43 & 50 & 100 \\
>10 kg & 75 & 90 & 64 & - \\
Abnormal xylose absorption & 83 & 57 & 53 & 86 \\
< $50 \%$ normal lower limit & 42 & 81 & 56 & - \\
Abnormal Schilling test & 92 & 52 & 52 & 92 \\
< $50 \%$ normal lower limit & 92 & 86 & 79 & - \\
\hline
\end{tabular}

were identified. Weight loss of less than $5 \mathrm{~kg}$ therefore had a negative predictive value of $100 \%$ for an eventual microbiological diagnosis, whereas weight loss of greater than $10 \mathrm{~kg}$ had a positive predictive value of $64 \%$ and a sensitivity of $75 \%$ (Table).

Abnormality of xylose absorption (whether or not severe), however, was less discriminatory an abnormal result had only a $53 \%$ positive predictive value for a final microbiological diagnosis and a normal result only an $86 \%$ negative predictive value. Severe abnormality of xylose absorption had a specificity of $80 \%$ associated with a sensitivity of only $42 \%$ (Table). The receiver operating characteristics curve constructed from the xylose absorption data indicated no better discrimination from any other given level of cut off.

A microbiological diagnosis was made in only one patient with a normal Schilling test but of 14 patients with a severely abnormal result, pathogens were found in 11 (including all five patients with cryptosporidial infecton, and two patients with only moderate weight loss and stool volume). A normal Schilling test therefore had a $92 \%$ negative predictive value for a final microbiological diagnosis. A severely abnormal Schilling test, however, had a sensitivity of $92 \%$ and a positive predictive value of $79 \%$ for subsequent microbiological diagnosis (Table). The receiver operating characteristics curve for Schilling test results indicated that a cut off point at $50 \%$ of the lower limit of normal (by chance) corresponded to the best combination of sensitivity and specificity achievable.

A barium follow through study was abnormal in only two patients who had cryptosporidial infection, appreciable weight loss, and severely abnormal Schilling test results. All endoscopies were macroscopically normal.

\section{TREATMENT AND OUTCOME}

Patients were treated on the basis of the results obtained, using specific antibiotic drugs for the bacterial infections (ampicillin for $S$ enteritidis, erythromycin for $C$ jejuni), metronidazole for giardiasis and amoebiasis, and high dose acyclovir for herpes simplex virus. In all patients relevant treatment eradicated the organism for the duration of microbiological follow up (more than two months) and stool volumes were reduced. The five patients with cryptosporidial diarrhoea were treated with a variety of antimicrobial agents without success, but starting them on zidovudine and opioid antidiarrhoeal drugs was associated with control of symptoms and considerably reduced stool volumes in two 
patients. The other three patients failed to respond and died within one month with continued watery diarrhoea. Three of the patients with cytomegaloviris colitis were treated (the fourth was terminally ill by the time of diagnosis). One responded to continuous ganciclovir, one to a single three week course of phosphonoformate but the last showed no response to antiviral treatment.

\section{SCORING SYSTEM}

Stool volume and estimated weight loss provided almost entirely concordant information; they are clearly not independent variables as far as their diagnostic potential is concerned. Since stool volume, despite being a valuable marker of disease activity and response to treatment, is more difficult to measure (particularly in outpatients) and proves relatively expendable diagnostically, we used estimated weight loss as the preferred variable, grouping patients between the three arbitrary bands of severity described above. Of the other variables considered, Schilling test result, with receiver operating characteristics curve-determined cut off at $5 \cdot 5 \%$ or less, showed most promise. Application of Bayes' theorem led to the allocation of the following scores: weight loss less than $5 \mathrm{~kg}$ : $+0 \cdot 19$; 5-10 kg: +0.04; more than $10 \mathrm{~kg}:-0.25$; Schilling result: more than $5 \cdot 5 \%$ : $+0 \cdot 17 ; 5 \cdot 5 \%$ or less: $-\mathbf{0} \cdot \mathbf{4 8}$. Fortunately, almost as effective is a simplified version where weight loss is scored with 2 points for more than $10 \mathrm{~kg}$ loss, 1 point for a loss of 5-10 kg, and 0 points for weight loss of less than $5 \mathrm{~kg}$, and where points are allocated for the Shilling result as follows: more than 5.5\%: 0 point; $5 \cdot 5 \%$ or less: 2 points. Retrospective application to the present patients (predictably) shows a $5 \%$ frequency of pathogen recognition for patients with scores of $0-2(n=19)$, whereas $90 \%$ of patients with a score of $4(n=10)$ had at least one pathogen identified.

\section{Discussion}

Microbiological examination of numerous stool samples is evidently the most useful diagnostic procedure, and this applies equally to the present difficult group as to the generality of HIV infected patients with diarrhoea. ${ }^{9}$ The present results, however, help to indicate the patient in whom it is reasonable to conclude that a specific diagnosis is unlikely to emerge however many samples are studied. The value of a specific diagnosis is not always obvious when no established treatment exists (for example in cryptosporidiosis), but valuable prognostic information may be obtained (several of the present patients were moved from HIV group IV A to group IV Cl or IV C2) and, increasingly, effective new treatments (for example for cytomegalovirus) are becoming available.

It is acknowledged that the infecting organisms identified may not necessarily have been relevant to the chronic diarrhoea - particularly the bacterial and amoebic infections - as these may have arisen acutely and been superimposed on (still) undiagnosed chronic diarrhoea thus explaining why they were not detected on initial investigation. There was, however, a good and long lasting (more than two months) remission from diarrhoea after antibiotic treatment. Stool electron microscopy in the routine evaluation of these patients may be thought valuable, although in a large series of stool samples from patients with AIDS and diarrhoea (unpublished observations), no viral particles were observed or other evidence provided for infection missed by other investigations. Electron microscopy of small bowel mucosa, however, is of considerable value in the diagnosis of microsporidial infection, which may be associated with malabsorption and altered small bowel histology but without evidence of the organism in conventional duodenal pinch biopsy specimens. In one recent study of patients similar to those considered here, more than a third had Microsporidia present at jejunal electron microscopy. ${ }^{10}$

Good evidence for malabsorption was found frequently in this group of patients, but although there has been speculation that HIV itself causes malabsorption, ${ }^{2}$ the present data argue for malabsorption being linked to secondary microbial infection in many patients. The normal serum vitamin $\mathrm{B}-12$ values is unsurprising given the relatively short total illness period in the patients concerned, but the site of the abnormality in the two positive barium follow through examinations and the normal folate studies suggest that the malabsorption is predominantly of ileal origin. Recent reports of possible relevance here indicate that there may be two forms of cryptosporidial infection, one where organisms are detected in stool only with difficulty but small bowel involvement and B-12 malabsorption are prominent, and the other where oocysts are readily identified in stool and the Schilling test is usually normal."

As we have shown in other contexts, ${ }^{12} 13$ barium radiology and endoscopy are only rarely helpful in patients with AIDS and will not often be indicated, but rectal biopsy is a useful early investigation' and remains the only route to diagnosis in some patients.

Based on the results presented here, the diagnostic yield of further microbiological samples in patients who have lost less than $5 \mathrm{~kg}$ in weight and have a normal Schilling test is extremely low. A putative scoring system is suggested but this clearly needs prospective evaluation in future patient groups.

We thank our patients and our many colleagues without whose assistance this work would not have been possible, but are particularly indebted to Drs Shanson, Harcourt-Webster, and Gleeson.

1 Antony MA, Brandt LJ, Klein RS, Bernstein LH. Infectious diarrhea in patients with AIDS. Dig Dis Sci 1988; 33: $1141-6$

2 Nelson JA, Wiley CA, Reynolds-Kohler C, Reese CE, Margaretten W, Levy JA. Human immunodeficiency virus detected in bowel epithelium from patients with gastrointestinal symptoms. Lancet 1988; i: 259-62.

3 Centers for Disease Control. Classification system for human T-lymphotropic virus type III/lymphadenopathy-associated T-lymphotropic virus type III/lymphaden
virus infections. MMWR 1986; 35: 334-9.

4 Garcia LS, Bruckner DA, Brewer TC, Shimizu RY. Techniques for the recovery and identification of cryptosporidium oocysts from stool specimens. F Clin Microbiol 1983; 18: 0ocysts

5 Mitchison DA, Allen BW, Manickavasagar D. Selective Kirchner medium in the culture of specimens other tha sputum for mycobacteria. $\mathcal{F}$ Clin Pathol 1983; 36: 1357-61.

6 Freedman ALS. Evaluating and comparing imaging tech nique: a review and classification of study designs. $\mathrm{Br} \mathcal{J}$ Radiol 1987; 60: 1071-81. 
7 Griner PF, Mayewski RJ, Mushlin AI, Greenland P. Selection and interpretation of diagnostic tests and procedures: principles and applications. Ann Intern Med 1981; 94: $553-600$

8 Knill-Jones RP. Diagnostic systems as an aid to clinical decision making. BrMed F 1987; 295: 1392-6.

9 Connolly GM, Shanson D, Hawkins DA, Harcourt-Webster JN, Gazzard BG. Non-cryptosporidial diarrhoea in human immunodeficiency virus (HIV) infected patients. Gut 1989; 30: $195-200$.

10 Orenstein J, Steinberg W, Chiang J, Smith P, Rotterdam H, Kotler D. Intestinal microsporidiosis as a cause of diarrhoea in AIDS. Vth International Conference on AIDS, Montreal, 1989: 209 (Abstract)

11 Heller TD, Tierney AR, Kotler D. Variable localization of intestinal cryptosporidiosis in AIDS. Vth International Conference on AIDS, Montreal, 1989: 358 (Abstract). 12 Connolly GM, Forbes A, Gleeson JA, Gazzard BG. InvestigaAIDS. $A I D S$ 1989; 3: 453-6.

13 Connolly GM, Forbes A, Gazzard BG. Value of barium enema and colonoscopy in HIV positive patients with diarrhoea. [Abstract] Gut 1989; 30: A735. 